\title{
Science and instrumentation update from the Palomar testbed interferometer
}

\section{Andrew F. Boden}

Andrew F. Boden, "Science and instrumentation update from the Palomar testbed interferometer," Proc. SPIE 5491, New Frontiers in Stellar Interferometry, (20 October 2004); doi: 10.1117/12.559988

Event: SPIE Astronomical Telescopes + Instrumentation, 2004, Glasgow, United Kingdom 


\title{
Science and Instrumentation Update from the Palomar Testbed Interferometer
}

\author{
A. Boden - Michelson Science Center/California Institute of Technology \\ (for the PTI Collaboration)
}

\begin{abstract}
The Palomar Testbed Interferometer (PTI) is a near-infrared, long-baseline interferometer located at the Palomar Observatory. PTI obtained first fringes in 1995, and has been in routine scientific operations since 1998.

PTI was primarily designed as a technology demonstration experiment for the Keck Interferometer, and has been successful in demonstrating 100-uas-class differential astrometry and two-combiner phase referencing. In addition to its engineering development accomplishments, PTI has been extraordinarily scientifically productive, producing more than 25 refereed scientific papers to date. This contribution will provide an update on PTI's operational, technical, and scientific status.
\end{abstract}

\section{Introduction}

The Palomar Testbed Interferometer (PTI) is a near-infrared (K and H-band), long-baseline interferometer located at Palomar Observatory in North San Diego County, California USA. PTI was conceived and designed as a technology development experiment for the Keck Interferometer (KI; Wizinowich 2004), specifically targeting the demonstration of dual-star narrow-angle astrometry, and phase referencing between two adjacent stars (Shao \& Colavita 1992). PTI obtained first fringes in 1995, and published its first peer-reviewed science paper in 1998. PTI has been in routine science operations since mid-1997, and to date has published over 25 peer-reviewed science papers. At the time of this writing, we expect to continue PTI science operations through 2005.

A detailed PTI instrument description can be found in Colavita et al (1999). Like most optical/near-infrared interferometers, PTI is a direct-detection interferometer, performing delay compensation and beam combination in freespace. PTI performs pairwise combination between three siderostat placements, providing three operational baselines on the order of 100m in length (PTI_NS - 110m, PTI_NW - 85m, and PTI_SW - 87m), making PTI's typical K-band fringe spacings between 4 and 5 milliarcseconds for sources at the zenith. Further, like KI and NPOI, PTI is a phasecoherent, fringe tracking interferometer, resulting in high science data throughput. PTI is unique in that it has dual-star feeds, differential delay lines, and two beam combiners, allowing PTI to simultaneously track fringes on two independent stars. PTI's limiting K-band sensitivity is a $\mathrm{K} \sim 6.5$ for unresolved sources, and $\mathrm{K} \sim 6$ for resolved sources.

Following on from previous PTI status updates by Wallace et al (1998), Lane et al (2000), and Lane et al (2003), this contribution will provide an update on PTI's instrumentation, operations, and scientific status, and touch briefly on PTI's future plans.

\section{Instrumentation Update}

Driven by the requirements for demonstrating dual-star astrometry, PTI's unique instrumental attributes are dual-star feeds, differential delay lines, and dual beam combiners, allowing it to simultaneously track fringes on two adjacent stars. This capability serves dual-star astrometry by leveraging the atmospheric correlation between adjacent stars to greatly reduce the relative pathlength fluctuations and perform precision differential astrometry; results of these experiments have been separately reported by Shao et al (1999) and Lane (2000). 
Over the past two years the principle instrumental upgrade at PTI has been the addition of a phase-referenced fringe scanning mode. In this mode, one (primary) PTI beam combiner is used to track fringes on a target. The second (secondary) beam combiner is used to scan the fringe envelope of the primary target and/or a secondary target. Pathlength corrections from the primary beam combiner are fed to the common delay lines used to delay light to going to both the primary and secondary combiners, thus creating an artificially long coherence time for the secondary combination. This artificially long coherence time on the secondary combiner greatly improves the quality of the fringe envelope measurements; arguments for the improvements resulting from this phase referencing are presented in Lane \& Mutterspaugh (2004b, these proceedings).

This fringe-scanning mode can be used to support both double-Fourier narrow-band visibility measurements, and precise ultra-narrow angle astrometry. At the time of this writing PTI has demonstrated 20 microarcsecond-class differential astrometry between two stars in a $\sim 0.5$ arcsecond field (cite Lane \& Mutterspaugh 2004a, Lane \& Mutterspaugh 2004b, Mutterspaugh \& Lane 2004, see Figure 1). This capability opens the possibility for a compelling astrometric planet search program for "S-type" planets around components in binary star systems with typical apparent separations of 100s of milliarcseconds - this program will be discussed below.

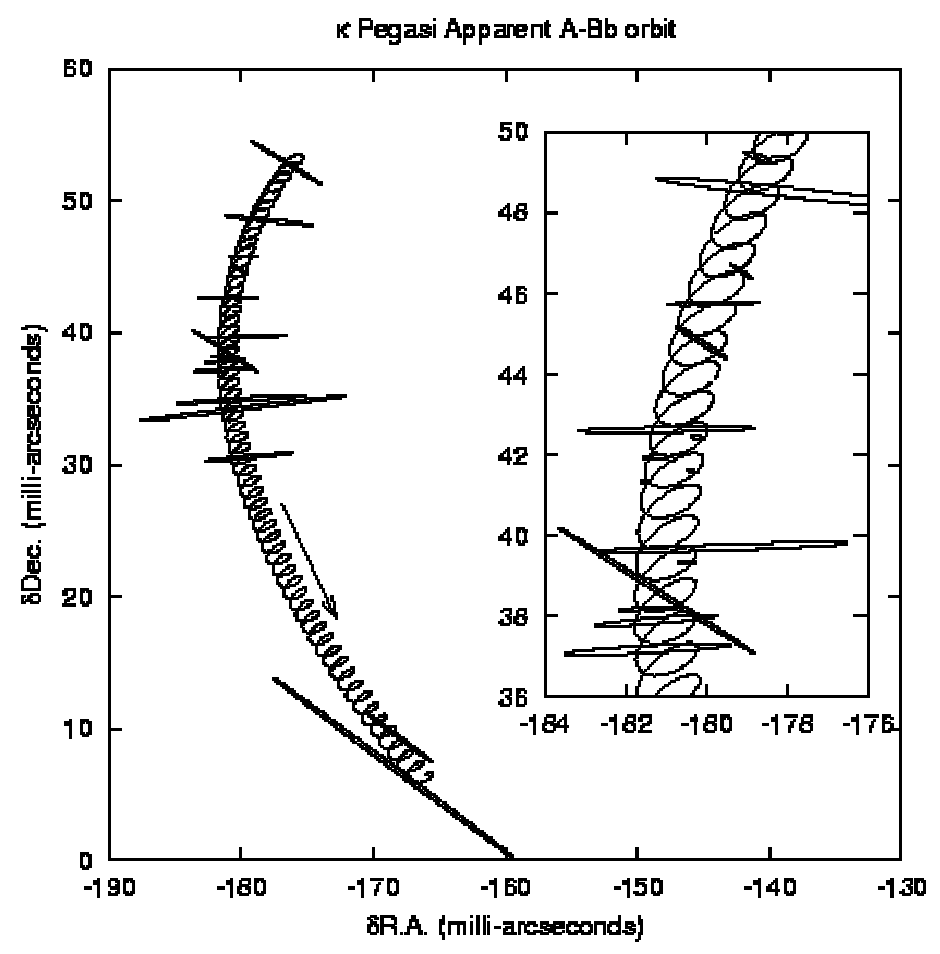

Figure 1: Relative Orbit of the Triple System $\kappa$ Pegasi (HD 206901) from PTI Fringe Scanning Astrometry (from Mutterspaugh \& Lane 2004).

Supporting the new fringe scanning mode is the addition of a new low-noise fringe camera in the secondary beam combiner. This camera is based on a PICNIC infrared array, and its sensitivity and noise performance is slightly better than the performance we see from our NICMOS-based camera on the primary table.

\section{Operations Update}


PTI continues to productively operate on a full-time basis, collecting data approximately $220-240$ nights in each of the 2002 and 2003 observing seasons, and on average making approximately 100 interferometric scans each night. PTI operates as a scientific collaboration (http://pti.jpl.nasa.gov), and is administered for the PTI collaboration and the greater JPL/Caltech community by the Michelson Science Center (MSC; $\underline{h t t p: / / m s c . c a l t e c h . e d u) . ~ P T I ~ o p e r a t e s ~ i n ~ a ~}$ queue-scheduled observing mode with the support of a full-time professional observer. Each year the PTI Time Allocation Committee (TAC) solicits observing proposals from members of the PTI Collaboration (http://pti.jpl.nasa.gov/ptimembers.html). PTI time allocations are made based on reviews of submitted proposals, and nightly observing schedules are composed based on these time allocations.

In addition to a number of smaller programs, in 2004 PTI selected two key project proposals aimed at providing for PTI scientific legacy. First, the PHASES (Palomar High-precision AStrometric Exoplanet Search - PI S Kulkarni) project aims to use the new fringe-scanning astrometric mode to conduct a survey for "S-type" planets around components in binary star systems with typical apparent separations of 100s of milliarcseconds. We anticipate the PHASES project will receive 80 PTI observing nights in each of the 2004 and 2005 observing seasons. Mutterspaugh and Lane (2004) give more details on the PHASES project. The second key project is the TPF Interferometric Target Survey (PI A. Boden), which is using PTI to survey candidate Terrestrial Planet Finder (TPF - see http://planetquest.jpl.nasa.gov/TPF/tpf index.html) target stars (the TPF candidate target list is maintained at http://sco.stsci.edu/tpf tldb). The objectives of this survey are to detect unknown or verify previously suspected multiplicity in these TPF candidate stars (e.g. see Figure 2), and in limited cases to constrain the apparent diameters of these stars. The Navy Prototype Optical Interferometer (NPOI) is conducting a similar survey in optical (see Hutter et al 2004), and the results of these two surveys will provide complementary databases to support final TPF target selection.

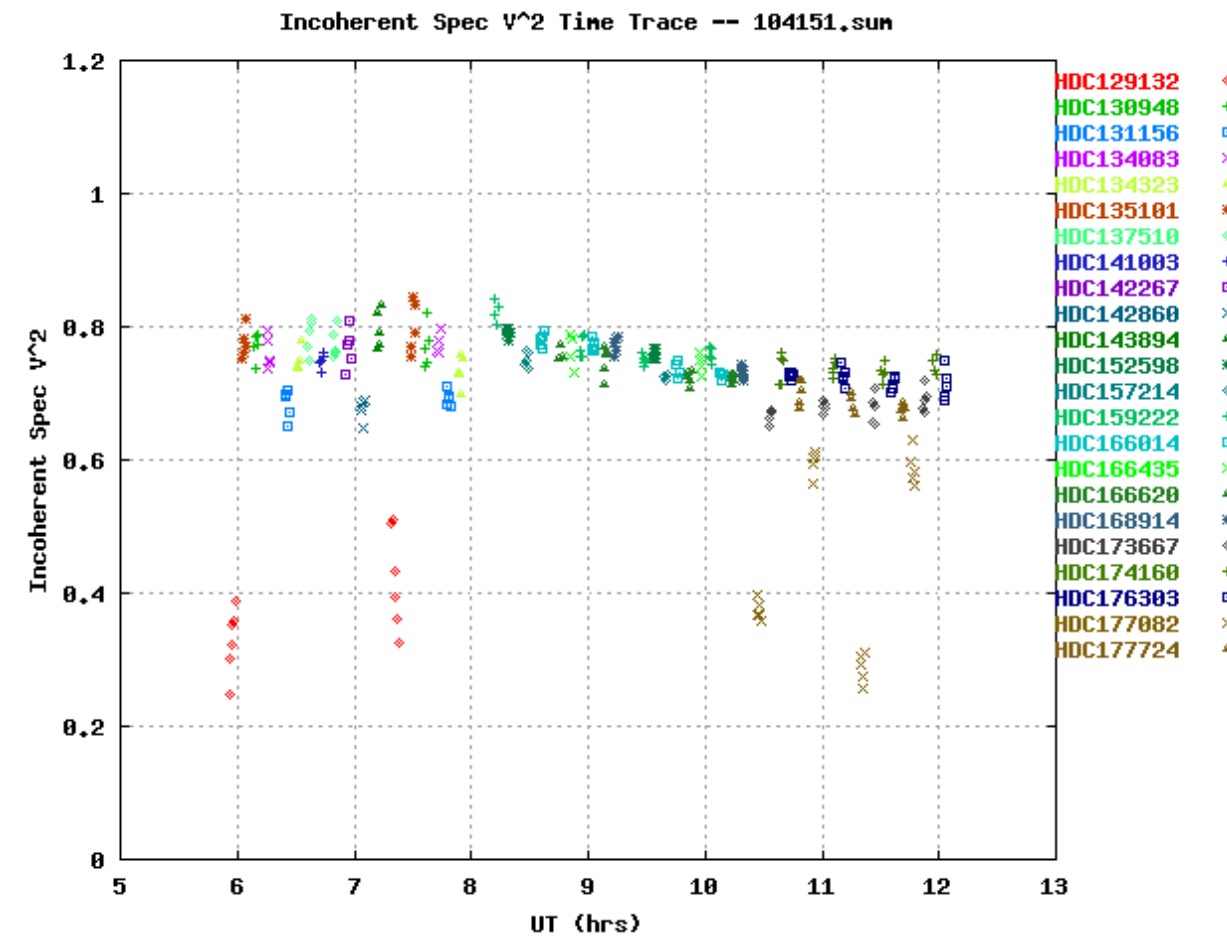

Figure 2. Confirmation "Image" for Binarity in the HD 177082 System. PTI visibility measurements on HD 177082 from night 104151 (20 May 2004 UTC) clearly indicate the system is resolved. Follow-up observations confirm this result. 
In addition to the high degree of computer automation for PTI real-time control, PTI science operations are supported by a mature set of observation planning tools (the getCal planning tool suite - see

http://msc.caltech.edu/software/getCal), data pipeline, and a posteriori data calibration tools (see

http://msc.caltech.edu/software/wbCalib and http://msc.caltech.edu/software/nbCalib).

\section{Science Update}

To date PTI has produced over 25 peer-reviewed scientific publications (a complete listing is available at http://pti.jpl.nasa.gov/publications.html), and over the past two years the PTI Collaboration has been scientifically active, producing a number of compelling new results. Some of the highlights from this reporting period are:

- Distance to the Pleiades inferred from the orbit of Atlas. Since the release of the Hipparcos catalog there has been a discrepancy between the distance estimates to the open cluster M45 (aka the Pleiades) made by Hipparcos and main-sequence fitting techniques (see Reid 1999 and van Leeuwen for cogent summaries of the issue). PTI visibility measurements were used to measure the visual orbit of the B-type binary Atlas (HD 23850) by Pan et al (2004), and by inferring the system mass based on stellar models estimated the Atlas system (and by consequence the Pleiades) distance at $135+/-3 \mathrm{pc}$. Counterarguments against the Pan et al result were offered by Perryman (2004 - see http://www.rssd.esa.int/hipparcos/Pleiades distance.html) based in part on the use of a single binary system to infer the Pleiades distance, however a recent analysis of the double-lined eclipsing binary system HD 23642 by Munari et al (2004) estimates the distance to a second binary in excellent agreement with the Atlas result.

- Studies of Circumstellar Material in Pre-Main Sequence Stars. PTI, IOTA (Traub 2004), and KI (Wizinowich 2004) have all been active in studying the near-infrared emission from pre-main sequence stars over the past several years. PTI has recently contributed new observations of T Tauri stars (Akeson et al 2002) and Herbig Ae/Be stars (Eisner et al 2003).

- Stellar Astrophysics: PTI continues to make useful measurements of fundamental stellar properties such as mass, luminosity, and apparent diameter. Mass and luminosity parameters are primarily accessible through studies of binary systems: Torres et al (2002) and Konacki \& Lane (2004) report studies of new binary systems based on PTI observations. Lane et al (2002) have continued their studies of Cepheid variable stars. And Thompson et al (2002a and 2002b) have studied various aspects of Mira variable stars with PTI observations.

\section{Summary and Future Plans}

The PTI Collaboration is justifiably proud of the accomplishments of PTI as a facility and an organization. In addition to demonstrating key technologies for KI development, operations, and analysis, PTI has been extraordinarily scientifically productive. PTI is in its eighth year of normal science operations, and during that period PTI has produced data on nearly 1300 nights. At the time of this writing it is our plan to operate PTI through the 2004 and 2005 observing seasons. Beyond that time we are yet to make a decision as to whether PTI shall continue operating into 2006.

The large dataset that PTI has amassed is a considerable scientific legacy. Because this dataset represents a valuable community resource, the PTI collaboration and the MSC are making plans to make the PTI dataset openly available to the astronomical community. Further details on the availability of PTI data to the general community will be forthcoming.

\section{Acknowledgements}

The author gratefully acknowledges the greater PTI Collaboration for support and camaraderie over the past eight years. In particular it is appropriate to acknowledge Mike Shao and Mark Colavita, who had the vision and fortitude to bring PTI into existence. The collaboration also gratefully acknowledges the invaluable contributions of our professional observer Kevin Rykoski. PTI is administered by the Michelson Science Center, and the collaboration acknowledges the continuing support of MSC Directors past and present. Finally, the PTI Collaboration gratefully acknowledges the support of NASA in the development and operations of PTI. 
This work has made use of services from the Michelson Science Center, California Institute of Technology, http://msc.caltech.edu. This work has also made use of the SIMBAD database, operated at CDS, Strasbourg, France.

\section{References}

1. Akeson et al 2002, "Constraints on Circumstellar Disk Parameters from Multi-wavelength Observations: T Tau and SU Aur", ApJ 566, 1124.

2. Colavita et al 1999, "The Palomar Testbed Interferometer", ApJ 510, 505.

3. Eisner et al 2003, "Near-Infrared Interferometric Measurements of Herbig Ae/Be Stars", ApJ 588, 360.

4. Konacki \& Lane 2004, "The Visual Orbits of the Spectroscopic Binaries HD 6118 and HD 27483 from the Palomar Testbed Interferometer", ApJ 610, 443.

5. Lane \& Colavita 2004, "Phase Referenced Stellar Interferometry at the Palomar Testbed Interferometer", AJ, $125,1623$.

6. Lane et al 2000, "Palomar Testbed Interferometer: update", Proc SPIE 4006, 452

7. Lane et al 2002, "Long baseline interferometric observations of Cepheids", ApJ, 573, 330.

8. Lane et al 2003, "Palomar testbed interferometer status report", Proc. SPIE, 4838, 62.

9. Lane \& Mutterspaugh 2004a, "Differential Astrometry of Sub-arcsecond Scale Binaries at the Palomar Testbed Interferometer", ApJ 601, 1129.

10. Lane \& Mutterspaugh 2004b, "Phase referencing and narrow-angle astrometry in current and future interferometers" SPIE 5491-06 (these proceedings)

11. Munari et al 2004, "The distance to the Pleiades from orbital solution of the double-lined eclipsing binary HD 23642", A\&A 418, L31.

12. Mutterspaugh \& Lane 2004, "Initial scientific results from phase-referenced astrometry on sub-arcsecond binaries", SPIE 5491-10 (these proceedings)

13. Pan, Shao, \& Kulkarni 2004, "A Distance of 133-137 Parsecs to the Pleiades Star Cluster", Nature 427, 326.

14. Reid 1999, "The HR Diagram and the Galactic Distance Scale After HIPPARCOS" ARAA 37, 191.

15. Shao \& Colavita 1992, "Potential of long-baseline infrared interferometry for narrow-angle astrometry", A\&A 262, 353.

16. Shao et al 1999, "Differential Astrometry of the 61 Cygni System with the Palomar Testbed Interferometer", BAAS 195, 8714

17. Thompson et al 2002a, "Time-Dependent Asymmetries in the Atmosphere of the Mira Variable R Tri Through IR Interferometry", ApJ 570, 373.

18. Thompson et al 2002b, "Multi-Epoch Interferometric Study of Mira Variables I. Narrowband Diameters of RZ Peg and S Lac" ApJ 577, 447.

19. Traub et al 2004, "IOTA: Recent technology and science", SPIE 5491-54 (these proceedings).

20. van Leeuwen 1999, "HIPPARCOS distance calibrations for 9 open clusters", A\&A 341, L71.

21. Wallace et al 1998, "Palomar Testbed Interferometer", Proc SPIE 3350, 864.

22. Wizinowich et al 2004, "Keck Interferometer status and plans", SPIE 5491-51 (these proceedings). 